University of Nebraska - Lincoln

DigitalCommons@University of Nebraska - Lincoln

Papers in Plant Pathology

Plant Pathology Department

March 2007

\title{
Functional characterization of the Aspergillus fumigatus calcineurin
}

\author{
Márcia Eliana da Silva Ferreira \\ Faculdade de Ciências Farmacêuticas de Ribeirão, Universidade de São Paulo, Brazil \\ Thorsten Heinekamp \\ Department of Molecular and Applied Microbiology, Leibniz Institute for Natural Product Research and \\ Infection Biology, Hans-Knoell-Institute (HKI), Germany \\ Albert Hartl \\ Drug Testing Group, Leibniz Institute for Natural Product Research and Infection Biology, Hans-Knoell- \\ Institute (HKI), Germany \\ Axel A. Brakhage \\ Department of Molecular and Applied Microbiology, Leibniz Institute for Natural Product Research and \\ Infection Biology, Hans-Knoell-Institute (HKI), Germany \\ Camile P. Semighini \\ University of Nebraska-Lincoln \\ See next page for additional authors \\ Follow this and additional works at: https://digitalcommons.unl.edu/plantpathpapers \\ Part of the Plant Pathology Commons
}

da Silva Ferreira, Márcia Eliana ; Heinekamp, Thorsten; Hartl, Albert; Brakhage, Axel A.; Semighini, Camile P.; Harris, Steven D.; Savoldi, Marcela; Fagundes de Gouvêa, Paula ; de Souza Goldman, Maria Helena; and Goldman, Gustavo H., "Functional characterization of the Aspergillus fumigatus calcineurin" (2007).

Papers in Plant Pathology. 87.

https://digitalcommons.unl.edu/plantpathpapers/87

This Article is brought to you for free and open access by the Plant Pathology Department at DigitalCommons@University of Nebraska - Lincoln. It has been accepted for inclusion in Papers in Plant Pathology by an authorized administrator of DigitalCommons@University of Nebraska - Lincoln. 


\section{Authors}

Márcia Eliana da Silva Ferreira, Thorsten Heinekamp, Albert Hartl, Axel A. Brakhage, Camile P. Semighini, Steven D. Harris, Marcela Savoldi, Paula Fagundes de Gouvêa, Maria Helena de Souza Goldman, and Gustavo H. Goldman 
Published in Fungal Genetics and Biology, Volume 44, Issue 3 (March 2007), pp. 219-230; doi:10.1016/j.fgb.2006.08.004 Copyright (C) 2007

Elsevier Inc. Used by permission. http://www.sciencedirect.com/science/journal/10871845

Submitted April 18, 2006; accepted August 10, 2006; published online September 20, 2006.

\title{
Functional characterization of the Aspergillus fumigatus calcineurin
}

\author{
Márcia Eliana da Silva Ferreira, ${ }^{\mathrm{a}}$ Thorsten Heinekamp, ${ }^{\mathrm{b}}$ Albert Härtl, ${ }^{\mathrm{c}}$ Axel A. Brakhage, ${ }^{\mathrm{b}}$ \\ Camile P. Semighini, ${ }^{\mathrm{d}}$ Steven D. Harris, ${ }^{\mathrm{d}}$ Marcela Savoldi, ${ }^{\mathrm{a}}$ Paula Fagundes de Gouvêa, ${ }^{\mathrm{a}}$ \\ Maria Helena de Souza Goldman, ${ }^{\mathrm{e}}$ and Gustavo H. Goldman ${ }^{\mathrm{a}, *}$ \\ ${ }^{a}$ Faculdade de Ciências Farmacêuticas de Ribeirão, Universidade de São Paulo, Brazil \\ ${ }^{\mathrm{b}}$ Department of Molecular and Applied Microbiology, Leibniz Institute for Natural Product Re- \\ search and Infection Biology, Hans-Knoell-Institute (HKI), Germany \\ ${ }^{\mathrm{c}}$ Drug Testing Group, Leibniz Institute for Natural Product Research and Infection Biology, \\ Hans-Knoell-Institute (HKI), Germany \\ d Plant Science Initiative, University of Nebraska-Lincoln, USA \\ ${ }^{\mathrm{e}}$ Faculdade de Filosofia, Ciências e Letras de Ribeirão Preto, Universidade de São Paulo, Brazil \\ * Corresponding author. Fax: +55 16633 1092; Email: ggoldman@usp.br (G.H. Goldman).
}

\begin{abstract}
Aspergillus fumigatus is an aggressive opportunistic pathogen of humans as well as a major allergen. Environmental sensing and retrieving essential nutrients from the environment are general metabolic traits associated with the growth of this saprophytic fungus. Two important mediators of calcium signals in eukaryotic cells are the $\mathrm{Ca}^{2+}$-binding protein calmodulin and the $\mathrm{Ca}^{2+}$ /calmodulin-dependent phosphatase calcineurin. Calcineurin is a heterodimer that consists of a catalytic subunit A and a $\mathrm{Ca}^{2+} /$ calmodulin binding unit. We deleted the A. fumigatus calA gene, which encodes the calcineurin A catalytic subunit, and demonstrated that this gene is not essential in this fungus. The $\triangle$ calA mutant strain has severe defects in growth extension, branching and conidial architecture. Furthermore, the A. fumigatus $\Delta$ calA mutant strain has decreased fitness in a low dose murine infection and cannot grow in fetal bovine serum (FBS). After potassium phosphate was added to liquid FBS, the $\triangle$ calA mutant strain could grow with the characteristic phenotype of the $\triangle$ calA mutation. When $A$. fumigatus calcineurin is inhibited by tacrolimus in a phosphate depleted medium, there is a reduction in the inorganic phosphate transport and six putative phosphate transporter genes have altered mRNA levels. However, there is no effect on the acid phosphatase activity. These results suggest that calcineurin is involved in the regulation of the $P H O$ pathway in $A$. $f u$ migatus. Our work on calcineurin opens new venues for the research on sensing and nutrient acquisition in A. fumigatus.
\end{abstract}

Keywords: Aspergillus fumigatus, Calcineurin, Phosphate assimilation

\section{Introduction}

Aspergillus fumigatus is a primary and opportunistic pathogen as well as a major allergen (Denning, 1998, Casadevall and Pirofski, 1999 and Greenberger, 2002). Because $A$. fumigatus produces prodigious amounts of conidia, the human respiratory tract is continuously exposed to the fungus (Latge, 1999). This fungus is increasingly linked to severe asthma and sinusitis (Latge, 1999). A. fumigatus is isolated from human habitats and vegetable compost heaps (Beffa et al., 1998 and Latge, 1999). The incidence of invasive infection in im- munocompromised individuals can be as high as $50 \%$ and the mortality rate is often $\sim 50 \%$ (Denning, 1998 and Brakhage, 2005). In spite of its great medical and ecological importance, the basic biology of the organism is not very well understood. However, the recent completion of the A. fumigatus genome sequence (Nierman et al., 2005) has offered an opportunity to investigate complete pathways and classes of genes and to elucidate its main biological mechanisms.

Environmental sensing and the retrieval of essential nutrients from the environment are general metabolic traits that are associated with the growth of the saprophyte A. fumigatus 
in inhospitable environments (Tekaia and Latgé, 2005). Calcium is a ubiquitous second messenger that functions in signal transduction pathways in eukaryotic organisms. Calcium signaling is important for several cellular processes, including fertilization and development, exocytosis, muscle contraction, motility, chemotaxis, cell division, differentiation, programmed cell death and chromatin remodeling in multicellular eukaryotes (Carafoli, 2005). In filamentous fungi, $\mathrm{Ca}^{2+}$ is important for the regulation of hyphal morphogenesis, i.e., hyphal tip growth events involving branching and orientation (Jackson and Heath, 1993, Fox and Heitman, 2002 and Kraus and Heitman, 2003). Two important mediators of calcium signals in eukaryotic cells are the $\mathrm{Ca}^{2+}$-binding protein calmodulin and the $\mathrm{Ca}^{2+} /$ calmodulin-dependent phosphatase calcineurin (Carafoli, 2005). Calcineurin is a serine/threonine-specific protein phosphatase heterodimer consisting of a catalytic subunit $\mathrm{A}$ and $\mathrm{a} \mathrm{Ca}^{2+} /$ calmodulin binding unit. The association of the two subunits is essential for activity (Fox and Heitman, 2002). Calcineurin is required for signal transduction such as the activation of T cells (Schreiber and Crabtree, 1992). Calcineurin signaling is inhibited by the immunosuppressive drugs cyclosporin A (CsA) and FK506 (tacrolimus) that bind to the immunophilins cyclophilin and FKBP12, respectively (Liu et al., 1991). The cyclophilin-CsA and FKBP12-FK506 complexes then inhibit calcineurin (Liu et al., 1991). Recent investigations into the molecular mechanisms of pathogenesis in $C$. albicans, $C$. neoformans, and A. fumigatus have revealed an essential role for calcineurin in morphogenesis, virulence and antifungal drug action (Cruz et al., 2001a, Cruz et al., 2001b, Fox and Heitman, 2002, Kontonyannis et al., 2003, Steinbach et al., 2004a and Steinbach et al., 2004b). In C. neofomans, calcineurin is essential for growth at $37^{\circ} \mathrm{C}$ but not at ambient temperature, and is also required for hyphal elongation during mating and haploid fruiting (Odom et al., 1997, Cruz et al., 2000, Cruz et al., 2001a and Fox et al., 2001). C. albicans calcineurin mutants had reduced virulence in a murine model of systemic infection (Cruz et al., 2002 and Sanglard et al., 2003). C. albicans calcineurin mutant strains failed to colonize and grow in kidneys of infected animals and were unable to survive when exposed to serum in vitro (Blankenship et al., 2003). The immunosupressants CsA, FK506, and sirolimus (formerly called rapamycin) exhibit in vitro and in vivo activity against $C$. albicans and $C$. neoformans, including fungicidal synergy with azole antifungals (Cruz et al., 2001b). It has been reported that therapy with calcineurin pathway inhibitors and existing antifungal agents augment activity against $A$. fumigatus (Kontonyannis et al., 2003, Steinbach et al., 2004a and Steinbach et al., 2004b).

We have previously deleted the calA gene, which encodes the calcineurin A catalytic subunit CalA (Ferreira et al., 2006). The $\Delta$ calA strain produced small sporulating colonies that showed comparable growth at different temperatures and increased branching when compared to the corresponding wild-type strain (Ferreira et al., 2006). Here, we extended our previous studies by showing that $A$. fumigatus $\triangle c a l A$ mutant strain has severe defects in growth extension. Furthermore, the $\triangle$ calA mutant strain has decreased fitness in a low dose murine infection and cannot grow in fetal bovine serum. Our data suggest a novel relationship between calcineurin function and inorganic phosphate acquisition in A. fumigatus.

\section{Materials and methods}

\subsection{Strains and media methods}

Aspergillus fumigatus strains used are CEA17 $\Delta \mathrm{ak}-$ uBKU80 (wild-type), $\triangle$ calA, and $\triangle$ calA $c a l A^{+}$. Media were of two basic types. A complete medium with three variants: YAG $(2 \%$ glucose, $0.5 \%$ yeast extract, $2 \%$ agar, trace elements), YUU (YAG supplemented with $1.2 \mathrm{~g} / \mathrm{l}$ each of uracil and uridine) and liquid $\mathrm{YG}$ or $\mathrm{YG}+\mathrm{UU}$ medium of the same compositions (but without agar). A modified minimal medium (MM: $1 \%$ glucose, original high nitrate salts, trace elements, $2 \%$ agar, $\mathrm{pH} 6.5$ ); or minimal medium without $\mathrm{KH}_{2} \mathrm{PO}_{4}$ (PF$\mathrm{MM}$ ). Trace elements, vitamins, and nitrate salts are described by Kafer (1977; Appendix, available on request from the author). Additionally, 10\% fetal bovine serum (Gibco) was used as a medium. CsA, tacrolimus, and sirolimus are Neoral ${ }^{\mathrm{R}} \mathrm{San}-$ dimmun (from Novartis), Prograf (from Janssen-Cilag), and Rapamune (from Wyeth), respectively.

For the disk diffusion assay, after standardized growth and harvest, $1.0 \times 10^{7} \mathrm{~A}$. fumigatus conidia were resuspended in $5 \mathrm{ml}$ of YAG top-agar medium and poured onto YAG agar plates. Blank 6-mm-diameter paper disks (3 MM, Whatman) were impregnated with $25 \mu \mathrm{g} / \mathrm{disk}, 100 \mu \mathrm{g} / \mathrm{disk}$, and $10 \mu \mathrm{g} /$ disk of tacrolimus, cyclosporin, and sirolimus, respectively, in YAG. The plates were incubated at $37^{\circ} \mathrm{C}$, and the diameters of the inhibition zones were evaluated after 24 or $48 \mathrm{~h}$. Each disk diffusion assay was performed twice.

The viability of growing hyphae was measured as described by Bruschi et al. (2001). A suspension of conidiospores (approximately $10^{6}$ conidia) was spread onto a $\mathrm{MM}$ plate using sterile glass spreader, and incubated for $16 \mathrm{~h}$ at $37^{\circ} \mathrm{C}$ to produce a nonsporulating mycelial "mat." Mycelial "plugs" were cut from the mats using the wide end of a sterile Pasteur pipette. The plugs (four samples of each mutant) were transferred onto MM plates. The diameters of the resulting colonies were measured and radial growth was expressed in $\mathrm{cm}$.

Phosphate transport in the wild-type and $\Delta$ calA strains was measured by ${ }^{32} \mathrm{P}$-labelled orthophosphate. Freshly harvested conidia of wild-type strain was inoculated into $10 \mathrm{ml}$ of liquid MM medium $\left(1 \times 10^{7}\right.$ conidia $\left./ \mathrm{ml}\right)$ and grown for $16 \mathrm{~h}$ at $37^{\circ} \mathrm{C}$. Mycelia were aseptically transferred to $5 \mathrm{ml}$ of liquid PF-MM free medium plus $1.0 \mu \mathrm{Ci}{ }^{32} \mathrm{P}$-labelled orthophosphate (Amersham Biosciences, UK) in the presence and absence of $2 \mu \mathrm{g} / \mathrm{ml}$ of tacrolimus, and incubated at $37^{\circ} \mathrm{C}$ for 2,4 , and $6 \mathrm{~h}$. The mycelia were washed at least 
four times with water, dried, weighted, and then phosphate incorporation was measured. The counts per minute (cpm) for triplicate samples were averaged. The same experimental design was repeated for wild-type and $\triangle$ calA mutant strains phosphate incorporation.

\subsection{Acid phosphatase enzymatic assay}

Conidia $\left(5 \times 10^{7} / \mathrm{ml}\right)$ were inoculated in $250-\mathrm{ml} \mathrm{Er-}$ lenmeyer flasks with $50 \mathrm{ml}$ of $\mathrm{MM}$ and incubated at $37^{\circ} \mathrm{C} / 150 \mathrm{rpm}$ for $16 \mathrm{~h}$. Mycelia were filtered, washed with sterile water and transferred to 250-ml Erlenmeyer flasks with $50 \mathrm{ml}$ of PF-MM. These cultures were subsequently incubated at $37^{\circ} \mathrm{C} / 150 \mathrm{rpm}$ for 1,2 , and $4 \mathrm{~h}$. One hundred microliters of each supernatant were added to a mixture reaction of $510 \mu \mathrm{l}(250 \mu \mathrm{l}$ sodium acetate buffer $100 \mathrm{mM} \mathrm{pH} 4.6$, $10 \mu \mathrm{l}$ of $\mathrm{MgCl}_{2} 100 \mathrm{mM}, 250 \mu \mathrm{l}$ of $p$-nitro phenyl phosphate $10 \mathrm{mM}$ ), that was incubated at $37^{\circ} \mathrm{C}$ for $60 \mathrm{~min}$. After this period of time, the reaction was terminated by adding $1 \mathrm{ml}$ of $\mathrm{NaOH} 1 \mathrm{M}$. The results were expressed as nanomole of $p$-nitrophenol liberated/mg of mycelial dry weigh/min (Caddick and Arst, 1986).

\subsection{DNA manipulations and construction of the AcalA strain}

DNA manipulations were according to Sambrook et al. (1989). DNA fragment probes for Southern blots were labeled with $\left[{ }^{32} \mathrm{P}-\alpha\right] \mathrm{dCTP}$ using the Random Primers DNA labeling System kit (Invitrogen). PCR primers were designed for amplifying each DNA fragment necessary for PCR-mediated technique by using Primer Express Version 1.0 (Applied Biosystems) design software. For the construction of the deletion, the A. fumigatus pyrG gene was amplified from the plasmid pCDA21 (Chaveroche et al., 2000), and is referred as zeopyrG cassette because the amplified fragment also contains the zeocin-resistance gene. The PCR-mediated construction for the calA gene consisted of three initial amplifications that generated a 5'- and 3'-flanking region of calA gene and a final fusion PCR (for further details, see Ferreira et al., 2006). For the DNA fragments containing the flanking regions, genomic DNA was used as a template. The 5'-flanking fragment, which encompasses $2000 \mathrm{bp}$ upstream of the ATG start codon was amplified with the primers calcineurin 1 (5'-ggttcattgggaccagacc- $\left.3^{\prime}\right)$ and calcineurin 2 (5'-ctgagaattcccttgcgcagtgtgaactaag-3'). The $3^{\prime}$ flanking region, which encompasses 2000-bp downstream of the stop codon of the calA genomic sequence, was amplified with the primers calcineurin 3 (5'-tgaggcgaattcaattgcttcggtcacgtcca- $\left.3^{\prime}\right)$ and calcineurin 4 (5'-gcgaactctgaagctcggt-3'). The zeopyr $G$ cassette was amplified using plasmid pCDA2 1 as a template and the following primers: Calcin-ZeoF (5'-cacactgcgcaagggaattctcagtcctgctc- $\left.3^{\prime}\right)$ and Calcin-pyrG (5'-gaccgaagcaattgaattcgcctcaaacaatgc-3). The final 6400-bp fusion PCR fragment was generated using the three previous DNA fragments as templates and calcineurin 1 and calcineurin 4 as primers. The $50 \mu 1$ amplification mixture included $1 \mathrm{X}$ platinum Taq
DNA Polymerase High Fidelity buffer (Invitrogen), $3 \mathrm{mM}$ of $\mathrm{MgSO}_{4}, 20$ pmol of each primer, $0.4 \mathrm{mM}$ deoxynucleotide triphosphate (dNTP) mix, $1.0 \mathrm{U}$ of Taq DNA HiFi platinum polymerase (Invitrogen), and $500 \mathrm{ng}$ of genomic DNA or $100 \mathrm{ng}$ plasmid. PCR amplification was carried out in a PTC100 96-well thermal cycler (MJ Research), at $94{ }^{\circ} \mathrm{C}$ for $2 \mathrm{~min}$, and 30 times $94{ }^{\circ} \mathrm{C}$ for $1 \mathrm{~min}, 58-60{ }^{\circ} \mathrm{C}$ (depending on the fragment) for $1 \mathrm{~min}$, and $68^{\circ} \mathrm{C}$ for $7 \mathrm{~min}$, followed by an extension step at $68{ }^{\circ} \mathrm{C}$ for $7 \mathrm{~min}$. After the reaction, the PCR products were purified with a Qiagen PCR cleanup kit according to the manufacturer's instructions.

The $\triangle$ calA strain was complemented by co-transformation of a linear fragment amplified from genomic DNA by using primers calcineurin 1 and 4, and a derivative of plasmid pAN7-1 (pHAT $\alpha$; Herrera-Estrella et al., 1990), that contains the $h p h$ gene encoding the Escherichia coli hygromycin phosphotransferase and confers resistance to hygromycin. Transformation of A. fumigatus strain $\triangle$ calA was according to the procedure of Osmani et al. (1987) using $5 \mu \mathrm{g}$ of linear or circular DNA fragments. Transformants were scored for their ability to grow on YG medium or in YUU plus $1 \mathrm{M}$ sucrose, in the presence of $200 \mu \mathrm{g} / \mathrm{ml}$ of hygromycin (Sigma-Aldrich). Southern analysis demonstrated that the deletion cassette had integrated at the calA locus. The $\Delta$ calA calA ${ }^{+}$strain $(\Delta$ calA complemented with the calA gene) shows essentially the same behavior of the wild-type strain.

\subsection{Real-time PCRs}

All the reactions were performed using an ABI Prism Sequence Detection System (Perkin-Elmer Applied Biosystem, USA). The SYBR ${ }^{\circledR}$ Green PCR Master Mix was used for PCRs. The thermal cycling conditions comprised an initial step at $95^{\circ} \mathrm{C}$ for $10 \mathrm{~min}$, followed by 40 cycles at $95^{\circ} \mathrm{C}$ for $15 \mathrm{~s}$ and $60^{\circ} \mathrm{C}$ for $30 \mathrm{~s}$. The reactions and calculations were performed according to Semighini et al. (2002). The oligonucleotides used are described in Table 1.

\subsection{Virulence assays}

Virulence of different A. fumigatus strains was determined in a murine low dose model for invasive aspergillosis as described previously (Liebmann et al., 2004 and Ferreira et al., 2006). Survival of female BALB/c mice (Harlan Winkelmann, Borchen, Germany) infected intranasally with conidia of A. fumigatus wild-type, $\triangle$ calA $\mathrm{calA}^{+}$, and $\triangle$ calA strains were monitored. Mices were immunosuppressed by intraperitoneal injection of cyclophosphamide $(150 \mathrm{mg} / \mathrm{kg}$ body weight) every third day, starting on day-4 prior to infection. Additionally, a single dose of cortisone acetate (200 mg/kg body weight) was injected subcutaneously on day-1. On day 0 , mices were infected with $3 \times 10^{4}$ of viable freshly harvested $A$. fumigatus conidia. Animals were tested in cohorts of five animals in sterile aerated cages. Survival was monitored at least twice a day for a time period of 14 days and moribund animals were sacrificed. 
Table 1. List of primers used in this work for the real-time RT-PCRs

\begin{tabular}{ll} 
Primer & \multicolumn{1}{c}{ Sequences $^{\mathbf{a}}$} \\
634FL/663RU & 5'-GTCGTACAGAGCCTCGTTGTCG-3' \\
634FL & 5'-CGAGCCCTCTCCGTTCACCAGCT[FAM]G-3' \\
MS1-F & 5'-TGTTCGTGCCTATATGAC-3' \\
MS1-R & 5'-GCTTCTGGAGGACGGACTGG-3' \\
MS2-F & 5'-CCTCCATCTACCTCACCGT-3' \\
MS2-R & 5'-GAGTGGTCGAGACCGGCATAC-3' \\
MS3-F & 5'-CGGGCCGTGAATGCCTATGT-3' \\
MS3-R & 5'-TGGATGTACGACTTGAGCC-3' \\
MS4-F & 5'-TGAAGAGCGCACTGGAGT-3' \\
MS4-R & 5'-GGCTTCCCGAACACATGGAT-3' \\
MS5-F & 5'-TGTTGGCCTTATGTGTTTC-3' \\
MS5-R & 5'-CACGTCATTGGGAGCGTTGT-3' \\
MS6-F & 5'-GCCTTCGATGCTCTCATTCG-3' \\
MS6-R & 5'-CTGCCCAGTAGACACCATC-3' \\
MS7-F & 5'-GTGGAGACTGGTGGTGGGAGTT-3' \\
MS7-R & 5'-AGCACTGAGGGGATAATC-3' \\
MS8-F & 5'-GGATCACGGCGTCAAGTGGT-3' \\
MS8-R & 5'-GCTTGAACAGCAGAGGAC-3' \\
MS9-F & 5'-GCTGGCGCGGTAGATACTGA-3' \\
MS9-R & 5'-AATCACAAGGAACCACAC-3' \\
a 6FAM, 6-carboxyfluorescein. &
\end{tabular}

Gene

Afu1g10910

Afu4g03610

Afu3g03010

Afu1g04290

Afu5g01960

Afu6g07750

Afu2g10690

Afu4g09210

Afu $7 g 06350$

Afu8g01850

\subsection{Microscopy}

Conidiospores from wild-type, $\triangle \mathrm{calA} \mathrm{calA}^{+}$, and $\Delta$ calA strains mutant were germinated at $37^{\circ} \mathrm{C}$ on glass coverslips arrayed on the bottom of a plastic $100 \mathrm{~mm}$ Petri dish containing the appropriate growth medium. Coverslips with adherent hyphae were fixed and stained as previously described (Harris et al., 1994). Nuclei were visualized by staining with Hoechst 33258 or DAPI, and septa were stained using Calcofluor. After staining, coverslips were washed in sterile water and mounted in $n$-propyl gallate. Slides were viewed using an Olympus BX51 fluorescent microscope. Images were captured with a Photometrics CoolSnap HQ CCD camera (Roper Scientific), and processed using IPLab software (Scanalytics, Inc.) and Adobe PhotoShop 6.0. Confocal images were obtained with an Olympus FW500/BX61 confocal laser scanning microscope using the following laserlines: $405 \mathrm{~nm}$ for Hoechst 33,258 and $488 \mathrm{~nm}$ for FITC. Images were captured by direct acquisition with a $Z$ step of $1 \mu \mathrm{m}$. They were subsequently processed using Adobe PhotoShop 6.0.

For scanning electron microscopical analysis of conidia, strains were cultivated for three days on MM agar plates and conidia were sampled directly on double-sided adhesive tape. Microscopy was performed on a scanning electron microscope LEO-1450VP (Zeiss, Germany).

\section{Results}

\subsection{Aspergillus fumigatus calcineurin catalytic subunit inac- tivated mutant (AcalA) has severe defects in growth extension}

We had previously deleted the calA gene that encodes the calcineurin A catalytic subunit ( $\triangle$ calA strain; Ferreira et al., 2006). As observed before, primary transformants displayed a pronounced phenotypic defect, i.e., small sporulating colonies (Figure 1A, upper panel; Ferreira et al., 2006). The diameter of the wild-type and $\triangle$ calA colonies was measured daily after the transfer of mycelial plugs which all had about one $\mathrm{cm}$ of diameter (Figure 1A, lower left panel). Wild-type colonies radiate outwards and occupy the entire plate surface by 3 days (average diameter of $7.5 \mathrm{~cm}$ ). The $\Delta$ calA strain has undulating colonies that display limited growth (average diameter of $1.5 \mathrm{~cm}$ ). The wild-type strain presented the expected hyphal margin at the edge of the colony (Figure 1A, lower right panel). Three independent measurements revealed that these hyphae extended $6.3 \mathrm{~cm}$ (standard deviation, $S D=1.53$ ) after three days. By contrast, the hyphal margin of the $\Delta$ calA strain did not extend normally, resulting in a compact colony (Figure 1A, lower right panel). The morphology of the $\triangle$ calA germlings showed increased apical branching when compared to the corresponding wild-type strain (Figure 1B). Measurements of the number of branches show that $60 \%$ of wild-type hyphae possess at least one branch and $30 \%$ have two or more, compared to 45 and $50 \%$ of $\triangle$ calA hyphae in each of these respective categories (Figure 1B, lower panel). The $\triangle$ calA strain possessed normal microtubules; however, although the conidiophores are normal, only having smaller vesicles when compared to the wild-type (data not shown), the calcineurin mutation influenced conidial morphology (Figure 1, Figure 2 and Figure 3). Wild-type spores presented a uniform morphology and size, whereas $47.5 \%(n=200, S D=7.8$, two independent experiments) of $\triangle$ calA spores exhibited abnormal size (represented by arrows in Figure 1C) or "tear-shape" morphology (represented by double arrows in Figure 1C). These abnormal spores have no nucleus and do not germinate; however this is not due to any technical problems since wild-type resting conidia stained with Hoescht (Figure 1 and Figure 2B). 
A
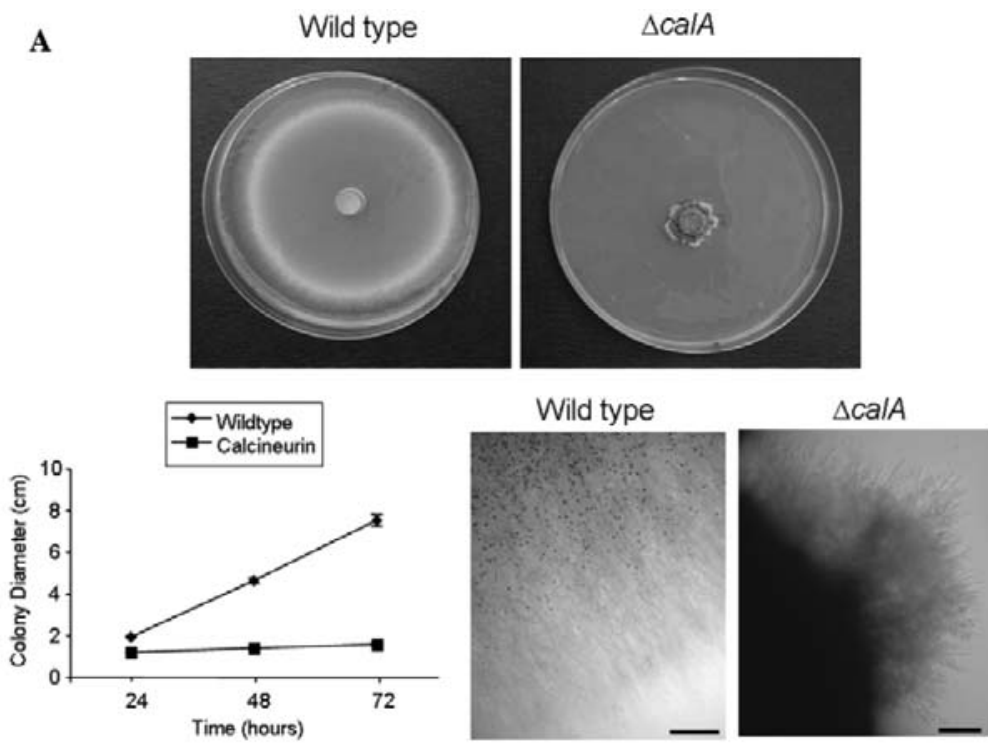

B
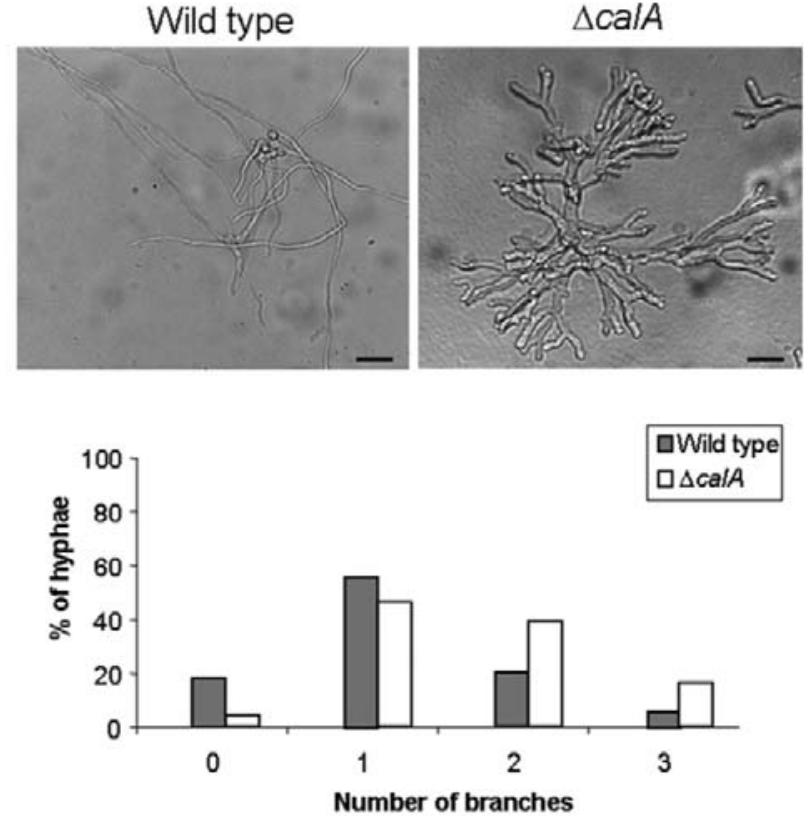

C
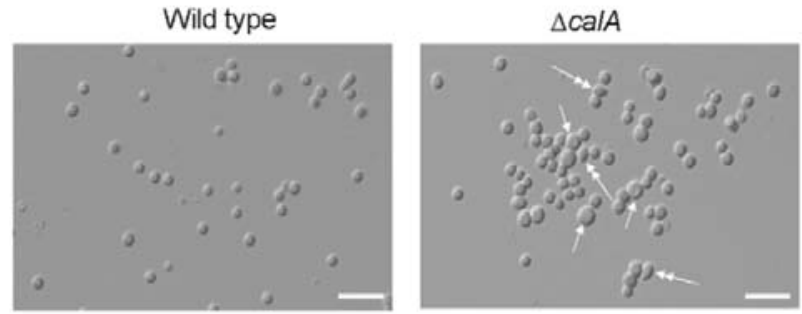

Figure 1. Calcineurin is required for colony extension: spores from each strain were inoculated in MM plates (plus supplements) and incubated at $37{ }^{\circ} \mathrm{C}$ overnight to produce a nonsporulating mycelial "mat." Mycelial "plugs" were cut from the mats using the wide end of a sterile Pasteur pipette. The plugs were transferred onto MM plates and incubated for 3 days at $37^{\circ} \mathrm{C}$. (A) Colony morphology (upper panels) and growth rate of wild-type (diamond shape) and $\Delta$ calA (square shape) colonies (lower left panel). The diameter of colonies was measured daily after the transfer of the mycelial plugs which were originally $1 \mathrm{~cm}$ in diameter. The average of three independent experiments is shown. Lower right panels are higher magnification images showing the edge of the colonies represented in the plates of the upper panels. Wild-type, bar, $100 \mu$ m; $\triangle c a l A$, bar, $10 \mu \mathrm{m}$. (B) Calcineurin is required for proper hyphal branching. Conidia of wild-type and $\Delta c a l A$ mutant strains were inoculated in liquid $\mathrm{YG}$ and incubated at $37^{\circ} \mathrm{C}$ for $10 \mathrm{~h}$ (upper panels; wild-type, bar, $27 \mu \mathrm{m} ; \Delta c a l A$, bar, $22 \mu \mathrm{m}$ ). Samples were fixed and stained with DAPI and calcofluor, then analyzed by light microscopy and the number of branches counted. (C) Calcineurin influences spore morphology. Wildtype spores display uniform morphology and size, whereas $\Delta$ calA spores (B) exhibit abnormal size (represented by arrows) or "tear-shape" morphology (represented by double arrows). Bars, $5 \mu \mathrm{m}$. Those abnormal spores have no nucleus. 

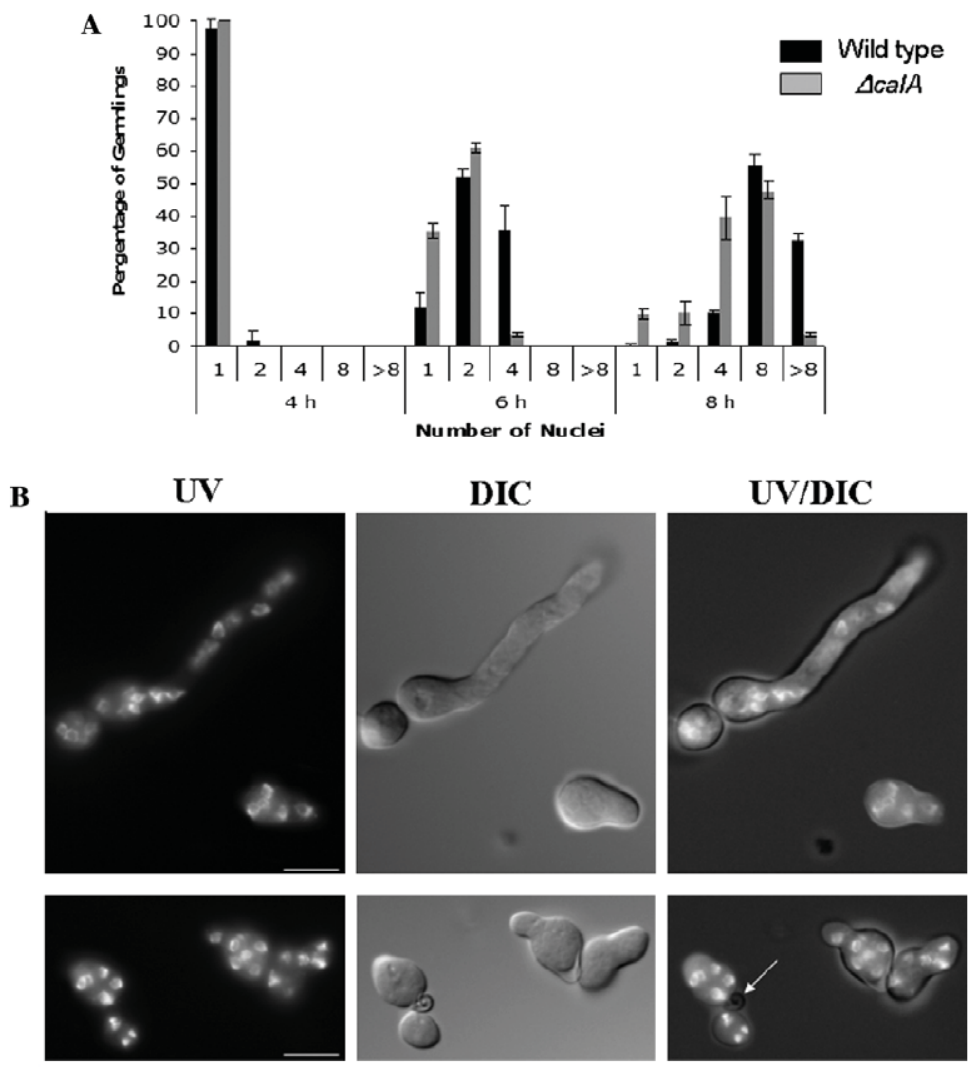

Figure 2. (A) Wild-type and mutant conidia were germinated on coverslips in proper liquid media for 4,6 , and $8 \mathrm{~h}$ at $37{ }^{\circ} \mathrm{C}$. Coverslips were fixed and stained with Hoechst 33258. Nuclei were visualized with fluorescence microscopy and the number of nuclei per germling was analyzed. For each sample, 200 germlings were examined in three independent experiments. (B) The wild-type and $\Delta c a l A$ mutant (lower panel) conidia germination after $8 \mathrm{~h}$. Note that the defective spore with tear shape (arrow) lacks nuclear staining, appears lysed and does not germinate. Bars, $10 \mu \mathrm{m}$ (UV and DIC are UV light and visible light fields, respectively).
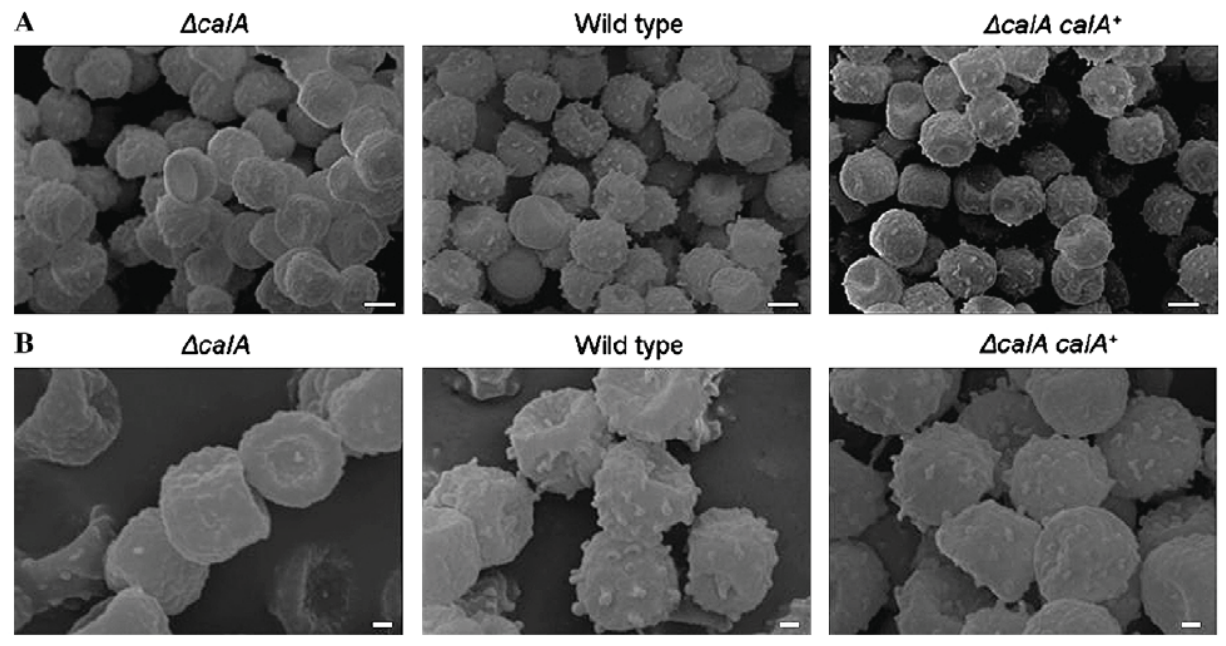

Figure 3. Conidia of the $\triangle$ calA mutant strain have reduced amounts of rodlets. Strains were cultivated for three days on MM agar plates and conidia were sampled directly on double-sided adhesive tape. Microscopy was performed on a scanning electron microscope LEO-1450VP (Zeiss, Germany). (A) 8000 times increase (bar, $300 \mathrm{~nm}$ ); (B) 16,000 times increase (bar, $1.0 \mu \mathrm{m}$ ).

The wild-type and $\triangle$ calA mutant strains display comparable nuclear division kinetics up to $4 \mathrm{~h}$ germination (Figure 2A). However, there is enhanced nuclear division kinetics in the wild-type strain with about 35 and $32 \%$ germlings having four and more than eight nuclei per germling at 6 and $8 \mathrm{~h}$ germination, respectively (Figure $2 \mathrm{~A}$ ). In contrast, the $\triangle$ calA mutant 


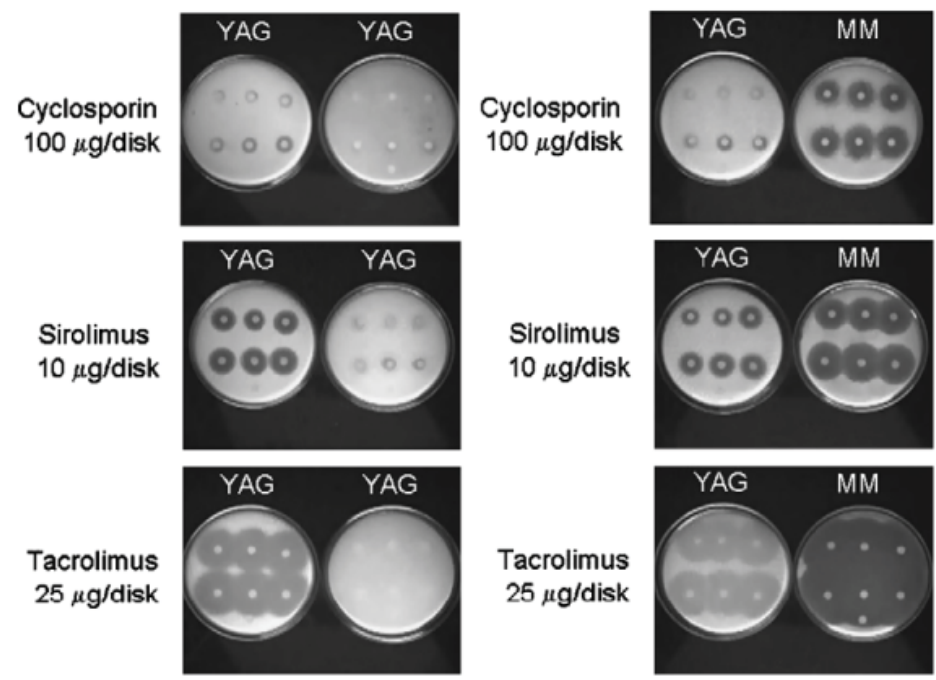

Figure 4. The $\triangle$ calA mutant strain shows resistance to immunosuppressants and the wild-type drug-sensitivity is increased in minimal medium. For disk diffusion assay, after standardized growth and harvest, $1.0 \times 10^{7}$ A. fumigatus conidia were resuspended in $5 \mathrm{ml}$ of YAG or MM topagar medium and poured onto YAG or MM agar plates. Blank 6-mm-diameter paper disks (3MM, Whatman) were impregnated with $25 \mu \mathrm{g} /$ disk, $100 \mu \mathrm{g} /$ disk, and $10 \mu \mathrm{g} /$ disk of tacrolimus, cyclosporin, and sirolimus, respectively. After drying, they were placed onto inoculated agar plates. The plates were incubated at $37^{\circ} \mathrm{C}$, and the diameters of the inhibition zones were evaluated after $24 \mathrm{~h}$. Each disk diffusion assay was performed twice and each plate has six disks repetitions of the same concentration of the specific immunosuppressor and a seventh disk containing the same volume of dimethyl sulfoxide that was used to dilute the immunosuppressor and was used as a negative control for the experiment. (A) The wild-type and $\triangle$ calA strains were inoculated in YAG medium plus different immunosuppressors. (B) The wild-type strain was inoculated in YAG and MM media plus different immunosuppressors.

strain has about 4 and 3\% germlings having four and more than eight nuclei per germling at 6 and $8 \mathrm{~h}$ germination, respectively (Figure 2A). We have not observed any effect of different temperatures, $\mathrm{pH}$ values, osmotic conditions, carbon sources, and increasing concentrations of Triton X-100, $\mathrm{CaCl}_{2}$, and $\mathrm{MnCl}$ on the growth of the $\triangle$ calA mutant strain (data not shown). These results suggest that the calA gene is not essential in A. fumigatus, but its deletion confers a severe growth defect and decreased rate of nuclear division kinetics.

Scanning electron microscopy revealed a morphological conidial defect in the $\triangle$ calA mutant (Figure 3). The conidia of wild-type A fumigatus are coated with hydrophobic proteins called rodlets which extend out from the conidial surface (Thau et al., 1994 and Girardin et al., 1999). The $\Delta$ calA mutant conidia seemed not to have these surface rodlets and instead contain smooth conidial surfaces (Figure 3).

Immunosuppressors such as tacrolimus (FK506) and CsA mediate their immunosuppressive effects with the peptidylprolyl isomerases, FKBP12 and cyclophilin A, respectively (Liu et al., 1991). Calcineurin is the target of the inhibitory FKBP12-FK506 and cyclophilin A-CsA complexes. CsA, tacrolimus, and sirolimus exhibit in vitro and in vivo activity against $A$. fumigatus (Steinbach et al., 2004a and Steinbach et al., 2004b). Thus, we tested the effect of the immunosupressants on $\triangle$ calA growth. As previously reported the A. fumigatus wild-type strain is sensitive to CsA, tacrolimus, and sirolimus (Kontonyannis et al., 2003, Steinbach et al., 2004a and Steinbach et al., 2004b; Figure 4A). However, the $\Delta$ calA strain displays almost no sensitivity to CsA and sirolimus, and no sensitivity at all to tacrolimus (Figure 4A). These results suggest that the $\triangle$ calA mutation confers increased resistance to immunosuppressors. We also decided to evaluate if the sensitivity of the wild-type strain to immunosuppressors could be modulated by the nutrient conditions. Thus, we grew the wildtype strain in YAG or MM in the presence of the immunosuppressants. As shown in Figure 4B, sensitivity to the immunosuppressants increased more than $50 \%$ when the wild-type strain was grown in MM. These results strongly indicate that calcineurin is important for growth in low nutrient conditions.

\subsection{Aspergillus fumigatus $\triangle$ calA mutant strain cannot grow in fetal bovine serum}

To determine a possible influence resulting from deletion of the calA gene, the corresponding deletion mutant was tested in an animal model. Virulence of A. fumigatus strain $\triangle$ calA in comparison to strains $\triangle$ calA $c a l A^{+}$, and wild-type, was determined in a murine low dose model for invasive aspergillosis as optimized by Liebmann et al. (2004). Considering the decreased viability of $\triangle$ calA conidia, mice were exposed to the same number of viable conidia for each strain $\left(3 \times 10^{4}\right.$ viable conidia). Infection with either the complemented $\Delta$ calA $\left(\triangle \mathrm{calA} \mathrm{calA}^{+}\right)$or the wild type strains resulted in a mortality rate of $70-90 \%$ after 10 days post inoculation of the conidia while the calA deletion strain resulted in a mortality rate of $20 \%$ (data not shown). These results indicate that the calA mutation is causing loss of fitness when the $\triangle$ calA strain is grown in the animal model when compared to the corresponding complemented and wild-type strains.

Next step, we tested the $\triangle$ calA mutant strain for growth in $10 \%$ FBS. Although the wild-type strain can grow in $10 \%$ FBS, the $\triangle$ calA strain cannot (Figure 5A). Since the $\Delta$ calA 
strain can grow in MM to the same extent as in YAG medium (data not shown), we hypothesize that a component of the MM would be able to complement the FBS growth defect from the $\triangle$ calA strain. The MM is composed of glucose, trace elements, and salt solution which were individually added to $10 \%$ FBS medium, and only the salt solution had obvious effects on the growth of the calcineurin mutant in $10 \%$ FBS (Figure 5A). The salt solution is composed of sodium nitrate, potassium chloride, potassium phosphate, and magnesium sulphate; only $11 \mathrm{mM}$ potassium phosphate could partially suppress the growth defect of the $\triangle$ calA mutant strain (Figure 5A). Even the wild-type can grow faster when potassium phosphate was added to $10 \%$ FBS medium (Figure 5A). When potassium phosphate was added to liquid $10 \%$ FBS, the $\triangle$ calA mutant strain grew and displayed the characteristic phenotype of the $\triangle$ calA mutation (Figure 5B). The $\triangle$ calA $\mathrm{calA}^{+}$strain showed the same behavior of the wild-type strain (data not shown). Since only potassium phosphate, but not potassium chloride, could suppress the absence of growth of the $\triangle$ calA mutant strain, we conclude that the calA gene is essential for the utilization of inorganic phosphate $\left(\mathrm{P}_{\mathrm{i}}\right)$.

In Saccharomyces cerevisiae, as a response to $\mathrm{P}_{\mathrm{i}}$ limitation, at least 22 PHO-related genes are derepressed, leading to elevated transcript levels, as judged by DNA microarray analysis together with genetic and biochemical studies (for a review, see Persson et al., 2003). To monitor the status of the PHO pathway, the activity of acid phosphatase which is transcriptionally induced as a response to $\mathrm{P}_{\mathrm{i}}$ starvation is commonly used (Persson et al., 2003). Thus, we decided to verify if when the calcineurin function is restrained, A. fumigatus is still able to secrete acid phosphatase. First, wild-type strain was grown for $16 \mathrm{~h}$ in liquid $\mathrm{MM}$ and then mycelia were transferred to liquid PF-MM either in the presence or absence of tacrolimus, and acid phosphatase activity was measured after 1, 2, and $4 \mathrm{~h}$ (Table 2). A. fumigatus mycelia grown either in the absence or presence of tacrolimus displayed comparable levels of acid phosphatase activity (Table 2). These results strongly suggest that the A. fumigatus $P H O$ pathway is still active when the calcineurin is inhibited in phosphate-depleted medium.

The absence of growth in the FBS medium could be related to a decreased transport of inorganic phosphate into $A$. fumigatus cells. To investigate this hypothesis, we have grown the wild-type in liquid $\mathrm{MM}$, the mycelia were transferred to liquid PF-MM either in the presence or absence of a sub-inhibitory concentration of tacrolimus $(2 \mu \mathrm{g} / \mathrm{ml})$ plus inorganic ${ }^{32} \mathrm{P}$, grown for 2,4 , and $6 \mathrm{~h}$ at $37^{\circ} \mathrm{C}$, and then the phosphate incorporation was measured. There is a reduction of about $22 \%$ in the phosphate incorporation when calcineurin is inhibited (Table 3). We also measured the phosphate incorporation in the $\triangle$ calA mutant strain. There is a reduction of about $15 \%$ $(2 \mathrm{~h})$ to $35 \%(4$ and $6 \mathrm{~h}$ ) in the phosphate incorporation into the $\triangle$ calA mutant strain (Table 3 ).

At low external $\mathrm{P}_{\mathrm{i}}$ concentrations, the expression of genes encoding high-affinity transporter encoding genes such as $S$. cerevisiae PHO 44 is up-regulated; this occurs by binding of the
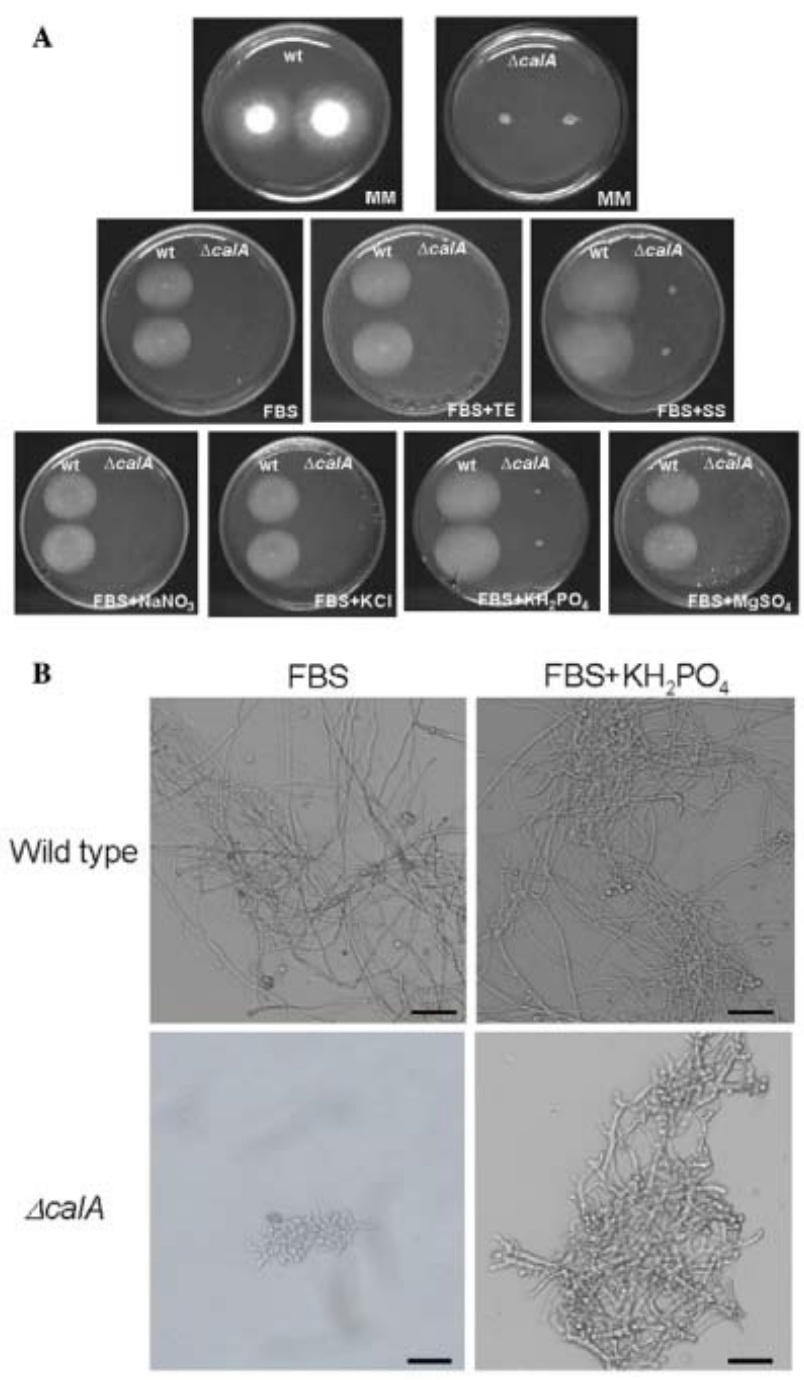

Figure 5. Aspergillus fumigatus $\triangle$ calA mutant cannot grow in fetal bovine serum without supplemented phosphate. (A) The wildtype and $\triangle$ calA mutant strains were point inoculated either in $\mathrm{MM}$ and grown for $48 \mathrm{~h}$ at $37^{\circ} \mathrm{C}$ (first row), or $10 \% \mathrm{FBS}, 10 \%$ FBS + TE (trace elements according to Kafer, 1977), and 10\% FBS + SS (salts solution according to Kafer, 1977) (second row), or $10 \% \mathrm{FBS}+\mathrm{NaNO}_{3}, 10 \% \mathrm{FBS}+\mathrm{KCL}, 10 \% \mathrm{FBS}+\mathrm{KH}_{2} \mathrm{PO}_{4}, 10 \%$ $\mathrm{FBS}+\mathrm{MgSO}_{4}$ (third row) and grown for $72 \mathrm{~h}$ at $37^{\circ} \mathrm{C}$. (B) Conidia from the wild-type and $\triangle$ calA mutant strains were inoculated in $10 \%$ FBS and FBS $+\mathrm{KH}_{2} \mathrm{PO}_{4}$ solid media and grown for $48 \mathrm{~h}$ at $37^{\circ} \mathrm{C}$. Bar, $33 \mu \mathrm{m}$. In both experiments in solid and liquid medium, the plates and tubes were incubated up to 10 days a $37^{\circ} \mathrm{C}$ and no growth was observed in the $\Delta$ calA mutant.

Table 2. Calcineurin inhibition does not affect the acid phosphatase activity $\mathrm{a}, \mathrm{b}$

$\begin{array}{llll} & \mathbf{1} \mathbf{h} & \mathbf{2} \mathbf{h} & \mathbf{4} \mathbf{h} \\ \text { PF-MM } & 305.0 \pm 49.5 & 338.0 \pm 69.7 & 478.6 \pm 50.6 \\ \text { PF-MM + Tacro } & 313.5 \pm 50.5 & 442.6 \pm 104.4 & 313.5 \pm 50.5\end{array}$

a Results are the average of three repetitions.

${ }^{\mathrm{b}}$ Acid phosphatase activity of culture filtrates expressed as nmol of $p$-nitrophenol liberated/mg of mycelial dry weight/min. 
Table 3. Inhibition of the calcineurin decreases the inorganic phosphate transport in A. fumigatus

$\begin{array}{lccc} & \mathbf{2} \mathbf{h}^{\mathbf{a}} & \mathbf{4} \mathbf{h} & \mathbf{6} \mathbf{~ h} \\ \text { Wild-type PF-MM } & 5.55 \pm 0.95 & 28.04 \pm 3.12 & 36.48 \pm 2.98 \\ \text { Wild-type PF-MM +TACRO } & 4.41 \pm 0.25 & 21.90 \pm 3.83 & 28.55 \pm 1.59 \\ \text { AcalA PF-MM } & 4.73 \pm 0.80 & 18.71 \pm 5.03 & 23.27 \pm 6.16 \\ & \\ \text { a Activity is expressed as } \times 10^{3} \mathrm{cpm} / \mathrm{mg} \text { dry weight and are the average of } \\ \text { three repetitions } \pm \text { standard deviation. }\end{array}$

Table 4. Expression of putative transporter encoding genes when $A$. fumigatus is grown in the presence of tacrolimus

$\begin{array}{lllcl}\text { Transporter genes }^{\mathbf{a}} & \mathbf{0} \mathbf{h}^{\mathbf{b}} & \mathbf{8} \mathbf{h} & \mathbf{8} \mathbf{h}+\text { TACRO } & \text { Changes (fold number) } \\ \text { Afu4g03610 } & 1.0 & 412 & 19 & \text { Decrease 22 X } \\ \text { Afu3g03010 } & 1.0 & 1,081 & 981 & \text { No change } \\ \text { Afu1g04290 } & 1.0 & 1.60 & 2.75 & \text { Increase 1.7 X } \\ \text { Afu5g01960 } & 1.0 & 1.79 & 2.74 & \text { Increase 1.53 X } \\ \text { Afu6g07750 } & 1.0 & 5.25 & 1.60 & \text { Decrease 3.30 X } \\ \text { Afu2g10690 } & 1.0 & 6.0 & 10.0 & \text { Increase 1.7 X } \\ \text { Afu4g09210 } & 1.0 & 10.6 & 13.0 & \text { No change } \\ \text { Afu7g06350 } & 1.0 & 259.6 & 217.5 & \text { No change } \\ \text { Afu8g01850 } & 1.0 & 8.5 & 34.7 & \text { Increase 4.1 X }\end{array}$

a The strains were grown for $16 \mathrm{~h}$ in $\mathrm{MM}$ and then transferred to PF-MM for $8 \mathrm{~h}$, in the presence and absence of $2 \mu \mathrm{g} / \mathrm{ml}$ of tacrolimus (TACRO) both at $37^{\circ} \mathrm{C}$

${ }^{\mathrm{b}}$ The measured quantity of the mRNA from the transporter genes in each of the treated samples was normalized using the $C_{\mathrm{t}}$ values obtained for the beta tubulin RNA amplifications run in the same plate. The relative quantitation of transporter and tubulin gene expression was determined by a standard curve (i.e., $C_{\mathrm{t}}$ values plotted against logarithm of the DNA copy number). Results of four sets of experiments were combined for each determination. The values represent the number of times the genes are expressed compared to the time zero control (represented absolutely as 1.00).

active transcription factors Pho4p and Pho2p to the promoter regions of the $\mathrm{PHO}$ genes (Persson et al., 2003). The $P H O$ pathway is poorly characterized in A. fumigatus, however we were able to identify nine genes encoding putative proteins that show high similarity to $S$. cerevisiae phosphate transporters (Blastp e-values $<1 \mathrm{e}-57$; $\geq 40 \%$ identity and $\geq 50 \%$ similarity; Afu4g03610, Afu3g03010, Afu1g04290, Afu6g07750, Afu4g09210, Afu2g10690, Afu7g06350, Afu8g01850, and Afu5g01960). Again, we used the same experimental strategy by inhibiting calcineurin function through the use of tacrolimus, and the expression of all these nine transporter encoding genes was assessed by using realtime RTPCR (Table 4). There are two genes that showed decreased mRNA accumulation when $A$. fumigatus was exposed to tacrolimus (Afu4g03610 and Afu6g07750). Interestingly, there are four genes that showed increased mRNA accumulation when calcineurin was inhibited (Afu1g04290, Afu5g01960, Afu2g10690, and Afu8g01850). The mRNA accumulation of three genes (Afu3g03010, Afu4g09210, and Afu 7g06350) was not significantly affected by growth in the presence of tacrolimus (Table 4).

Taken together these results suggest that there is a reduction in inorganic phosphate transport and mRNA accumula- tion of six putative phosphate transporter encoding genes is altered when calcineurin is inhibited in phosphate-depleted medium.

\section{Discussion}

Aspergillus fumigatus is a saprophytic fungus that is important for nutrient recycling in nature. However, A. fumigatus can also cause human disease as an opportunistic pathogen, and depending on the immune status of the patient, this fungus can elicit responses ranging from allergy to disseminated and frequently fatal infection. Sensing and nutrient acquisition mechanisms are essential for the fungus in order to discriminate in which environment it is growing. $A$. $f u$ migatus senses and responds to its immediate environment via signal transduction cascades, which transduce information from the outside environment to the inside the cell. One of these mechanisms of intracellular signaling includes $\mathrm{Ca}^{2+}$ ions, which bind to and activate calmodulin. The $\mathrm{Ca}^{2+}-$ calmodulin complex then binds to and activates calmodulin-dependent enzymes, such as the protein phosphatase calcineurin. Here, we show that calcineurin is not an essential gene in A. fumigatus, and present the roles of calcineurin in regulating differentiation and fitness in this opportunistic pathogen. In agreement to our results, Steinbach et al. (2006) have also recently shown that a mutant of $A$. fumigatus lacking the calcineurin A ( $c n a A)$ catalytic subunit exhibited defective hyphal morphology related to apical extension and polarized growth, which resulted in drastically decreased filamentation. Lung tissue from animals infected with the delta cnaA mutant showed a complete absence of hyphae, in contrast to tissue from animals infected with the wild-type and complemented strains.

\subsection{Calcineurin is essential for A. fumigatus colony extension}

Calcineurin plays an important role in the control of cell morphology and virulence in fungi. Growing hyphae contain a tip-high $\mathrm{Ca}^{2+}$ gradient thought to be crucial for establishing and maintaining apical organization, morphogenesis, and growth (Jackson and Heath, 1993). In N. crassa, the function of calcineurin was investigated by induction of antisense RNA expression and reduced levels of can-1 (encoding the catalytic subunit) mRNA and decreased calcineurin enzyme activity (Prokisch et al., 1997). Induction of the antisense RNA expression, caused growth arrest preceded by an increase in hyphal branching, changes in hyphal morphology and concomitant loss of the distinctive tip-high $\mathrm{Ca}^{2+}$ gradient typical for growing wild-type hyphae. In the same organism, the gene encoding the regulatory subunit $(c n b-1)$ was inactivated by an insertional mutagenesis procedure followed by a screening protocol designed to isolate mutants affected in the ability to regulate entry into conidiation (Kothe and Free, 1998). When $c n b-1$ was inactivated through RIP, the mutant showed a more severely restricted colonial growth pattern that consisted en- 
tirely of highly branched hyphae of swollen septated cells. It has been reported that calcineurin (cnaA) is an essential gene in A. nidulans, (Rasmussen et al., 1994). However, in contrast to these authors we have observed that the cnaA gene is not an essential gene and that the deletion strain displays a very closely related morphological phenotype to that seen for $A$. $f u$ migatus (Fagundes, unpublished results).

In C. neoformans, calcineurin is required for growth at $37^{\circ} \mathrm{C}$, virulence, and mating (Odom et al., 1997, Fox et al., 2001 and Cruz et al., 2001a). Calcineurin is not essential for the initial fusion event, but is required for hyphal elongation and survival of the heterokaryon produced by cell fusion (Cruz et al., 2001a). It is also required for hyphal elongation in diploid strains and during asexual haploid fruiting of MAT $\alpha$ cells in response to nitrogen limitation (Cruz et al., 2001a). Additionally, calcineurin regulates filamentation and $37^{\circ} \mathrm{C}$ growth via distinct mechanism. In $C$. albicans, deletion of the gene encoding the calcineurin A subunit (CNAI) affected colony morphology in several media known to modulate the $C$. albicans dimorphic switch (Sanglard et al., 2003).

We found that calcineurin is required for A. fumigatus colony extension and for normal conidial morphology. The absence of calcineurin also showed decreased nuclear division kinetics and increased branching in A. fumigatus. The $\Delta$ calA mutant conidia displayed abnormal morphology and are frequently anucleate. Analysis by scanning electronic microscopy showed that the surface of the conidia harvested from the $\triangle$ calA mutant have disorganized prominent protrusions. The interwoven proteinaceous microfibrils that decorate the outermost cell wall layer of Aspergillus conidia are called rodlets (Thau et al., 1994 and Girardin et al., 1999). Rodlets are composed of hydrophobins that confer physiochemical properties to mediate conidial dispersal, and possibly, cellular interactions (Thau et al., 1994 and Girardin et al., 1999). It is possible that the $\triangle$ calA mutant has decreased expression of hydrophobins and consequently decreased host tissue adhesion. Preliminary analysis in our laboratory indicates that $A$. fumigatus $\triangle$ calA mutant has decreased adhesion to laminin (data not shown).

\subsection{Calcineurin deletion mutant cannot grow in FBS}

We have shown that the A. fumigatus calcineurin deletion mutant has decreased virulence in a murine low dose model. Calcineurin is also important for virulence in C. albicans and C. neoformans (Cruz et al., 2001b, Bader et al., 2003 and Sanglard et al., 2003). However, the observed decreased virulence in the $\triangle$ calA mutant is most probably due to the decreased fitness of this strain. Furthermore, calcineurin is essential for A. fumigatus growth in bovine fetal serum, but growth can be rescued if inorganic phosphate is added to the serum, indicating that calcineurin is involved in phosphate acquisition in $10 \%$ FBS. It remains to be investigated if the FBS contains molecules that can chelate the free phosphate or inhibitors affecting phosphate uptake.
Phosphate is an ion that is essential for fungal growth and the amount of phosphate present in the serum is below $1 \mathrm{mM}$ (Tekaia and Latgé, 2005). Actually, the $\Delta$ calA mutant strain is also not able to grow in MM supplemented with inorganic phosphate in concentrations $\leq 1 \mathrm{mM}$ (data not shown). Cellular acquisition, storage and release, and metabolic integration of inorganic phosphate $(\mathrm{Pi})$ rely on the essential participation of numerous enzymes, such as extracellular acid phosphatases, phosphodiesterases, phosphate transporters, polyphosphate kinases, alkaline phosphatases, and endopolyphosphates (for a review, see Persson et al., 2003). In S. cerevisiae, the activities of the enzymes needed for intracellular Pi homeostasis are subjected to regulation via the Pi signal transduction pathway (PHO pathway) (Persson et al., 2003). In S. cerevisiae, a complex of cyclin-dependent kinase (CDK), Pho85p, one of its cyclins, Pho80p, and the CDK inhibitor (CKI), Pho81p, together with the transcription factors Pho4p and Pho2p constitute the core components of the transcriptional regulation of PHO genes (Persson et al., 2003).

To monitor the status of the $P H O$ pathway, the activity of secreted acid phosphatase (the gene product of $\mathrm{PHO}$, which is transcriptionally induced as a response to $P_{i}$ starvation) is commonly used (Persson et al., 2003). Thus, we investigated the activity levels of acid phosphatase when A. fumigatus wild-type is submitted or not to calcineurin inhibition by tacrolimus in PF-MM. Calcineurin inhibition does not affect the acid phosphatase activity, suggesting that the $P H O$ pathway is also active in the $\triangle$ calA mutant strain.

The systems for Pi transport have been characterized as a low-affinity (that assures a supply of Pi at normal or high external Pi concentrations) and a high-affinity (activated in response to Pi starvation) process (Persson et al., 1999). We have shown that there is a reduction in the inorganic phosphate transport when A. fumigatus calcineurin is inhibited. In $S$. cerevisiae, the genes are also regulated at the transcriptional level and we were able to show that the mRNA accumulation of some $A$. fumigatus putative phosphate transporter genes was altered when calcineurin is inhibited. These results suggest that the deficiency of phosphate acquisition could be due to decreased phosphate transport. The influence of calcineurin on phosphate transport has already been observed in the renal type II Na/Pi co-transporter (Moz et al., 2004). Mice with genetic deletion of the calcineurin $\mathrm{A} \beta$ gene had a marked decrease in type II Na/Pi mRNA levels and remarkably did not show the expected increase in type II Na/Pi mRNA levels after the challenge of a low-Pi diet. Recently, Sopko et al. (2006) identified the yeast calcineurin-responsive transcription factor Crzlp as a substrate for Pho85p, providing additional evidence for a link between the $P H O$ pathway and calcineurin regulation.

Calcineurin is also essential for C. albicans survival in serum (Blankenship et al., 2003 and Sanglard et al., 2003). However, unlike A. fumigatus, it has been proposed that the absence of growth is due to (i) the fact that in the serum lacks one or more components necessary for the proliferation of the 
mutant strain, and (ii) the presence of an active killing component in the serum. In C. albicans, addition of yeast extract or supraphysiologic levels of the vitamins thiamine and niacin rescued the growth of calcineurin mutants (Blankenship et al., 2003). Apparently, the mechanisms that underlie the absence of growth in serum in both biological systems could be different. However, we cannot completely discard the possibility that FBS contains another "inhibitory" factor that has a larger effect on growth of the already impaired mutant.

In summary, we have found that calcineurin is important for A. fumigatus morphogenesis, fitness, and growth in serum. The lost of fitness in the animal model and the inability to grow in FBS could be related to a combination of factors, such as decreased conidia and hyphae adhesion to the host tissue, and the nutritional deficiency to inorganic phosphate. Our work on calcineurin opens new venues for the research on sensing and nutrient acquisition in A. fumigatus.

\section{Acknowledgments}

This research was supported by the Fundação de Amparo à Pesquisa do Estado de São Paulo (FAPESP), Conselho Nacional de Desenvolvimento Científico e Tecnológico (CNPq), Brazil, and the Deutsche Forschungsgemeinschaft (SPP1160), Germany. We also thank Walter Richter and Renate Kaiser for helpful assistance in scanning electron microscopy, and the three anonymous reviewers for their comments.

\section{References}

Bader et al., 2003 - T. Bader, B. Bodendorfer, K. Schroppel and J. Morschhauser, Calcineurin is essential for virulence in Candida albicans, Infect. Immun. 71 (2003), pp. 5344-5354.

Beffa et al., 1998 - T. Beffa, F. Staib, J. Lott Fischer, P.F. Lyon, P. Gumowski, O.E. Marfenina, S. Dunoyer-Geindre, F. Georgen, R. Roch-Susuki, L. Gallaz and J.P. Latge, Mycological control and surveillance of biological waste and compost, Med. Mycol. 36 (1998) (Suppl. 1), pp. 137-145. Cited By in Scopus (0)

Blankenship et al., 2003- J.R. Blankenship, F.L. Wormley, M.K. Boyce, W.A. Schell, S.G. Filler, J.R. Perfect and J. Heitman, Calcineurin is essential for Candida albicans survival in serum and virulence, Eukaryot. Cell 2 (2003), pp. 422-430.

Brakhage, 2005- A.A. Brakhage, Systemic fungal infections caused by Aspergillus species: epidemiology, infection process and virulence determinants, Curr. Drug Targets 6 (2005), pp. $875-886$.

Bruschi et al., 2001- G.C.M. Bruschi, C.C. de Souza, M.R.Z.K. Fagundes, M.A.C. Dani, M.H.S. Goldman, N.R. Morris, L. Liu and G.H. Goldman, Sensitivity to camptothecin in Aspergillus nidulans identifies a novel gene, scaA, related to the cellular DNA damage response, Mol. Genet. Genom. 265 (2001), pp. 264-275.

Caddick and Arst, 1986- M.X. Caddick and H.N. Arst, Structural genes for phosphatases in Aspergillus nidulans, Genet. Res. Camb. 47 (1986), pp. 83-91.

Carafoli, 2005- E. Carafoli, Calcium - a universal carrier of biological signals, FEBS J. 272 (2005), pp. 1073-1089.

Casadevall and Pirofski, 1999- A. Casadevall and L.A. Pirofski, Host-pathogen interactions: redefining the basic concepts of vir- ulence and pathogenicity, Infect. Immun. 67 (1999), pp. 37033713.

Chaveroche et al., 2000- M.K. Chaveroche, J.M. Ghigo and C. d'Enfert, A rapid method for efficient gene replacement in the filamentous fungus Aspergillus nidulans, Nucleic Acids Res. 28 (2000), pp. E97-E104.

Cruz et al., 2000- M.C. Cruz, R.A. Sia, M. Olson, G.M. Cox and J. Heitman, Comparison of the roles of calcineurin in physiology and virulence in serotype D and serotype A strains of Cryptococcus neoformans, Infect. Immun. 68 (2000), pp. 982-985.

Cruz et al., 2001a - M.C. Cruz, D.S. Fox and J. Heitman, Calcineurin is required for hyphal elongationduring mating and haploid fruiting in Cryptococcus neoformans, EMBO J. 20 (2001), pp. 1020-1032.

Cruz et al., 2001b- M.C. Cruz, A.L. Goldstein, J. Blankenship, M. Del Poeta, J.R. Perfect, J.H. McCusker, Y.L. Bennani, M.E. Cardenas and J. Heitman, Rapamycin and less immunosuppressive analogs are toxic to Candida albicans and Cryptococcus neoformans via FKBP12-dependent inhibition of TOR, Antimicrob. Agents Chemother. 45 (2001), pp. 3162-3170.

Cruz et al., 2002- M.C. Cruz, A.L. Goldstein, J.R. Blankenship, M. Del Poeta, D. Davis, M.E. Cardenas, J.R. Perfect, J.H. McCusker and J. Heitman, Calcineurin is essential for survival during membrane stress in Candida albicans, EMBO J. 21 (2002), pp. 546-559.

Denning, 1998 - D.W. Denning, Invasive aspergillosis, Clin. Infect. Dis. 26 (1998), pp. 781-803.

Ferreira et al., 2006- M.E.S. Ferreira, M.R.V.Z. Kress, M. Savoldi, M.H.S. Goldman, A. Härtl, T. Heinekamp, A.A. Brakhage and G.H. Goldman, The $a k u B^{\mathrm{KU} 80}$ mutant deficient for nonhomologous end joining is a powerful tool for analyzing pathogenicity in Aspergillus fumigatus, Eukaryot. Cell 5 (2006), pp. 207-211.

Fox and Heitman, 2002 - D.S. Fox and J. Heitman, Good fungi gone bad: the corruption of calcineurin, BioEssays 24 (2002), pp. 894-903.

Fox et al., 2001- D.S. Fox, M.C. Cruz, R.A. Sia, H. Ke, G.M. Cox, M.E. Cardenas and J. Heitman, Calcineurin regulatory subunit is essential for virulence and mediates interactions with FKBP12FK506 in Cryptococcus neoformans, Mol. Microbiol. 39 (2001), pp. 835-849.

Girardin et al., 1999- H. Girardin, S. Paris, J. Rault, M.N. BellonFontaine and J.P. Latgé, The role of the rodlet structure on the physiochemical properties of Aspergillus conidia, Lett. Appl. Microbiol. 29 (1999), pp. 364-369.

Greenberger, 2002 - P.A. Greenberger, Allergic bronchopulmonary aspergillosis, J. Allergy Clin. Immunol. 110 (2002), pp. 685-692.

Harris et al., 1994 - S.D. Harris, J.L. Morrell and J.E. Hamer, Identification and characterization of Aspergillus nidulans mutants defective in cytokinesis, Genetics 136 (1994), pp. 517-532.

Herrera-Estrella et al., 1990 - A. Herrera-Estrella, G.H. Goldman and M. Van Montagu, High-efficiency transformation system for the biocontrol agents, Trichoderma spp, Mol. Microbiol. 4 (1990), pp. 839-843.

Jackson and Heath, 1993 - S.L. Jackson and I.B. Heath, Roles of calcium ions in hyphal tip growth, Microbiol. Rev. 57 (1993), pp. 367-382.

Kafer, 1977- E. Kafer, Meiotic and mitotic recombination in Aspergilllus and its chromosomal aberrations, Adv. Genet. 19 (1977), pp. 33-131. 
Kontonyannis et al., 2003- D.P. Kontonyannis, R.E. Lewis, N. Osherov, N.D. Albert and G.S. May, Combination of caspofungin with inhibitors of the calcineurin pathway attenuates growth in vitro in Aspergillus species, J. Antimicrob. Chemother. 51 (2003), pp. 313-316.

Kothe and Free, 1998 - G.O. Kothe and S.J. Free, Calcineurin subunit B is required for normal vegetative growth in Neurospora crassa, Fungal Genet. Biol. 23 (1998), pp. 248-258.

Kraus and Heitman, 2003 - P.R. Kraus and J. Heitman, Coping with stress: calmodulin and calcineurin, Biochem. Biophys. Res. Commun. 311 (2003), pp. 1151-1157.

Latge, 1999- J.P. Latge, Aspergillus fumigatus and aspergillosis, Clin. Microbiol. Rev. 12 (1999), pp. 310-350.

Liebmann et al., 2004- B. Liebmann, T.W. Muhleisen, M. Muller, M. Hecht, G. Weidner, A. Braun, M. Brock and A.A. Brakhage, Deletion of the Aspergillus fumigatus lysine biosynthesis gene lys $F$ encoding homoaconitase leads to attenuated virulence in a low-dose mouse infection model of invasive aspergillosis, Arch. Microbiol. 181 (2004), pp. 378-383.

Liu et al., 1991 - Y. Liu, J.D. Farmer Jr., W.S. Lane, J. Friedamn, I. Weissman and S.L. Schreiber, Calcineurin is a common target of cyclophilin-cyclosporin A and FKBP-FK506 complexes, Cell 66 (1991), pp. 807-815.

Moz et al., 2004 - Y. Moz, R. Levi, V. Lavi-Moshayoff, K.B. Cox, J.D. Molkentin, J. Silver and T. Naveh-Many, Calcineurin A $\beta$ is central to the expression of the renal type II $\mathrm{Na} / \mathrm{Pi}$ co-transporter gene and to the regulation of renal phosphate transport, J. Am. Soc. Nephrol. 15 (2004), pp. 2972-2980.

Nierman et al., 2005- W. Nierman, A. Pain, M.J. Anderson, J. Wortman, H.S. Kim, J. Arroya, M. Berriman, K. Abe, D.B. Archer, C. Bermejo, J. Bennett, P. Bowyer, D. Chen, M. Collins, R. Coulsen, R. Davies, P.S. Dyer, M. Farman, N. Fedorova, T.V. Feldblyum, R. Fischer, N. Fosker, A. Fraser, J.L. Garcia, M.J. Garcia, A. Goble, G.H. Goldman, K. Gomi, S. Griffith-Jones, R. Gwilliam, B. Haas, H. Haas, D. Harris, H. Horiuchi, J. Huang, S. Humphrey, J. Jimenez, N. Keller, H. Khouri, K. Kitamoto, T. Kobayashi, R. Kulkarni, T. Kumagai, A. Lafton, J.P. Latgé, W. Li, A. Lord, C. Lu, W.H. Majoros, G.S. May, B.L. Miller, Y. Mohamoud, M. Molina, M. Monod, I. Mouyna, S. Mulligan, L. Murphy, S. O’Neil, I. Paulsen, M.A. Penalva, M. Pertea, C. Price, B.L. Pritchard, M.A. Quail, E. Rabbinowitsch, N. Rawlins, M.-A. Rajandream, U. Reichard, H. Renauld, G.D. Robson, S. Rodriguez de Cordoba, J.M. Rodriguez-Pena, J.M. Ronning, C.M. Rutter, S.S.L. Salzberg, M. Sanchez, J.C. Sanchez-Ferrero, D. Saunders, K. Seeger, R. Squares, S. Squares, M. Takeuchi, F. Tekaia, G. Turner, C.R. Vazquez de Aldana, J. Weidman, O. White, J. Woodward, J.-H. Yu, C. Fraser, J.E. Galagan, K. Asai, M. Machida, N. Hall, B. Barrell and D.W. Denning, Genomic sequence of the pathogenic and allergenic filamentous fungus Aspergillus fumigatus, Nature 438 (2005), pp. 1151-1156.

Odom et al., 1997- A. Odom, S. Muir, E. Lim, D.L. Toffaletti, J. Perfect and J. Heitman, Calcineurin is required for virulence of Cryptococcus neoformans, EMBO J. 16 (1997), pp. 2576-2589.

Osmani et al., 1987- S.A. Osmani, G.S. May and N.R. Morris, Regulation of the mRNA levels of $\operatorname{nim} A$, a gene required for the G2-M transition in Aspergillus nidulans, J. Cell Biol. 104 (1987), pp. 1495-1504.

Persson et al., 1999- B.L. Persson, J. Petterson, U. Fristedt, R. Weinander, A. Berhe and J. Pattison, Phosphate permeases of Saccharomyces cerevisiae: structure, function, and regulation, Biochim. Biophys. Acta 1422 (1999), pp. 255-272.
Persson et al., 2003- B.L. Persson, J.O. Lagerstedt, J.R. Pratt, J. Pattison-Granberg, K. Lundh, S. Shokrollahzadeh and F. Lundh, Regulation of phosphate acquisition in Saccharomyces cerevisiae, Curr. Genet. 43 (2003), pp. 225-244.

Prokisch et al., 1997- H. Prokisch, O. Yarden, M. Dieminger, M. Tropschug and I.B. Barthelmess, Impairment of calcineurin function in Neurospora crassa reveals its essential role in hyphal growth, morphology and maintenance of the apical $\mathrm{Ca}^{2+}$ gradient, Mol. Gen. Genet. 256 (1997), pp. 104-114.

Rasmussen et al., 1994 - C. Rasmussen, C. Garen, S. Brining, R.L. Kincaid, R.L. Means and A.R. Means, The calmodulin-dependent protein phosphatase catalytic subunit (calcineurin A) is an essential gene in Aspergillus nidulans, EMBO J. 13 (1994), pp. $2545-2552$.

Sambrook et al., 1989 - J. Sambrook, E.F. Fritsch and T. Maniatis, Molecular Cloning: A Laboratory Manual (Second ed), Cold Spring Harbor Laboratory Press, Cold Spring Harbor, NY (1989).

Sanglard et al., 2003 - D. Sanglard, F. Ischer, O. Marchetti, J. Entenza and J. Bille, Calcineurin A of Candida albicans: involvement in antifungal tolerance, cell morphogenesis, and virulence, Mol. Microbiol. 48 (2003), pp. 959-976.

Schreiber and Crabtree, 1992 - S.L. Schreiber and G.R. Crabtree, The mechanism of action of cyclosporine A and FK506, Immunol. Today 13 (1992), pp. 136-142.

Semighini et al., 2002 - C.P. Semighini, M. Marins, M.H.S. Goldman and G.H. Goldman, Quantitative analysis of the relative transcript levels of ABC transporter Atr genes in Aspergillus nidulans by real-time reverse Transcripition-PCR assay, Appl. Environ. Microbiol. 68 (2002), pp. 1351-1357.

Sopko et al., 2006 - R. Sopko, D. Huang, N. Preston, G. Chua, B. Papp, K. Kafadar, M. Snyder, S.G. Oliver, M. Cyert, T.R. Hughes, C. Boone and B. Andrews, Mapping pathways and phenotypes by systematic gene overexpression, Mol. Cell 21 (2006), pp. 319-330.

Steinbach et al., 2004a- W.J. Steinbach, N. Singh, J.L. Miller, D.K. Benjamin Jr., W.A. Schell, J. Heitman and J.R. Perfect, In vitro interactions between antifungals and immunosuppressants against Aspergillus fumigatus isolates from transplant and nontransplant patients, Antimicrob. Agents Chemother. 48 (2004), pp. 4922-4925.

Steinbach et al., 2004b - W.J. Steinbach, W.A. Schell, J.R. Blankenship, C. Onyewu, J. Heitman and J.R. Perfect, In vitro interactions between antifungals and immunosuppressants against $\mathrm{As}$ pergillus fumigatus, Antimicrob. Agents Chemother. 48 (2004), pp. 1664-1669.

Steinbach et al., 2006- W.J. Steinbach, R.A. Cramer Jr., B.Z. Perfect, Y.G. Asfaw, T.C. Sauer, L.K. Najvar, W.R. Kirkpatrick, T.F. Patterson, D.K. Benjamin Jr., J. Heitman and J.R. Perfect, Calcineurin controls growth, morphology, and pathogenicity in Aspergillus fumigatus, Eukaryot. Cell 5 (2006), pp. 1091-1103.

Tekaia and Latgé, 2005- F. Tekaia and J-P. Latgé, Aspergillus fumigatus: saprophyte or pathogen?, Curr. Opin. Microbiol. 8 (2005), pp. 1-8.

Thau et al., 1994- N. Thau, M. Monod, B. Crestani, C. Rolland, G. Tronchin, J.-P. Latgé and S. Paris, Rodletless mutants of Aspergillus fumigatus, Infect. Immun. 62 (1994), pp. 4380-4388. 